\title{
Cost Avoidance vs. Utility Bill Accounting - Explaining the Discrepancy Between Guaranteed Savings in ESPC Projects and Utility Bills
}

\author{
Satish Kumar, LBNL \\ Dale Sartor, LBNL
}

\section{Background}

Federal agencies often ask if Energy Savings Performance Contracts (ESPCs) result in the energy and cost savings projected during the project development phase. After investing in ESPCs, federal agencies expect a reduction in the total energy use and energy cost at the agency level. Such questions about the program are common when implementing an ESPC project. But is this a fair or accurate perception? More importantly, should the federal agencies evaluate the success or failure of ESPCs by comparing the utility costs before and after project implementation?

In fact, ESPC contracts employ measurement and verification (M\&V) protocols to measure and ensure kilowatt-hour or BTU savings at the project level. In most cases, the translation to energy cost savings is not based on actual utility rate structure, but a "contracted utility rate" that takes the existing utility rate at the time the contract is signed with a clause to escalate the utility rate by a fixed percentage for the duration of the contract. Reporting mechanisms, which advertise these savings in dollars, may imply an impact to budgets at a much higher level depending on actual utility rate structure.

FEMP has prepared the following analysis to explain why the utility bill reduction may not materialize, demonstrate its larger implication on agency's energy reduction goals, and advocate setting the right expectations at the outset to preempt the often asked question - why I am not seeing the savings in my utility bill?

\section{Lessons Learned From the Case Study Approach}

Most of the effort to date on evaluating the discrepancy between the energy savings as guaranteed by the ESPC project and utility bill has focused on the individual case study approach. This approach has been helpful in understanding and documenting the discrepancy between ESPC payments and utility bill savings, as demonstrated by various "savings verification" studies performed by FEMP for ESPC projects at Ellis Island and Fort Polk and for a UESC project at Fort Detrick. Findings from the three case studies converge on the following factors contributing to these discrepancies:

1. The $M \& V$ approach employed by ESPCs tends to isolate the energy efficiency measure before performing the measurements, savings analysis, and calculations. Further, in many cases energy savings attributed to the ESPC project is such a small fraction (less than 10 percent) of the utility bill, that a meaningful savings validation study becomes difficult and costly. 
2. In many cases a master meter serves the ESPC site, and there is no capability to submeter individual buildings where energy efficiency measures were implemented. Moreover, the energy consumption reporting procedure makes matching ESPC guaranteed savings with utility bills a challenging exercise; only a few people may have the knowledge that would help match the project with the meter report containing corresponding utility bill information.

3. Differences in utility rate structures were also cited as one of the main reasons for the discrepancy, since the utility rate in the contract is never the same as the actual rate paid by the agency. If the actual utility rate increase is higher than the "contracted utility rate" used to calculate projected energy savings, a negative perception may form because the total utility cost has increased. In reality, the dollar savings attributed to the ESPCs may be higher than the guaranteed amount.

4. Weather conditions may be different from those assumed by ESCOs to project the energy savings.

5. Mission changes may result in operating hour or personnel fluctuations at the facility.

6. Changes in the area of the facility being served by the ESPC may also come into play.

The case study approach is valuable in addressing site-specific concerns and identifying major factors that may cause discrepancy, particularly when the same contributing factors are found at a number of sites. Another major contribution of this approach has been the "Utility Bill Comparison With and Without ESPC Project," which stresses the value of ESPC projects and helps silence inquiries by creating an adjusted baseline that is compared against the actual utility bills during the performance period of the ESPC project.

\section{Lessons Learned from the Agency Level Approach}

While the case study approach is helpful, site-specific findings (which do not fully explain the discrepancy at the agency or department level to program oversight bodies) are difficult to generalize and apply at the agency level. In order to address the concerns regarding the ability of the ESPCs to help reduce energy intensity (in Btu/square foot) and energy cost at the agency level, a different approach was taken.

This broader analysis focused on matching the utility bills with ESPC savings information by tracking the actual utility bill during both the baseline and initial performance period of two years. An analysis of utility bills for all sites implementing ESPC projects was compared with the results of sites where no energy efficiency projects have been implemented during the same time period. The analysis plotted the annual ESPC guaranteed savings as a percentage of the utility bill for the year the contract was signed (unadjusted) against the ratio of the current energy cost to the baseline energy cost for each performance year. A linear regression model was developed from the ESPC project data to show a correlation between annual ESPC guaranteed savings as a percentage of the utility bill and the percent reduction in energy cost achieved. In parallel, energy use and cost data from sites where no energy efficiency projects had been implemented was also plotted for different years to figure out the "baseline creep" that takes place with the passage of time. The "linear regression fit" of the data showed an 
increase in energy use above the "frozen baseline" when no ESPC projects would have been implemented.

Key findings of the macro-level analysis are that load creep and utility cost increases are occurring EVERY YEAR at both ESPC sites and at sites where no significant energy efficiency projects have been implemented. Most sites have experienced utility cost increases of about 10 percent per year that cannot be explained by poor ESPC performance, and load creep accounts for 25 percent of energy cost increases. These factors are largely responsible for the discrepancy between the guaranteed energy savings in ESPCs and actual utility bills. Baseline adjustments should be made that account for load creep and increased utility costs when calculating savings from ESPC projects.

\section{Conclusions}

The recent FEMP study, "Evaluation of Super ESPCs Performance Reports," states "It is important to note that in general, these performance reports are not expected to compare the Super ESPC project's savings to the site's overall current energy use ${ }^{1}$. Tracking and reporting a site's actual utility's rates and overall energy use is not included in most ESPC projects. ESPC performance reports are not intended to address the question "If I'm saving energy, why don't I see it in my utility bills?” Although this is a commonly asked question, the information required to answer this question is usually not available to the ESCO and is generally outside the scope of an ESPC project. Often, a site energy manager or a consultant is required to address this issue by providing a more comprehensive look at a site's overall use of energy."

While ESPC projects should always save dollars for federal facilities, they may not always reduce the utility bill (both energy use and dollars) for several reasons. Based on the experience and findings of this analysis, it is prudent not to associate the term "utility bill reduction" with ESPC projects, and instead use the term "cost avoidance" when discussing the benefits of implementing ESPC projects.

For more information, please contact Satish Kumar, LBNL, 202-646-7953 or SKumar@lbl.gov or Dale Sartor, LBNL, 510-486-5988 or DASartor@lbl.gov.

\footnotetext{
${ }^{1}$ Of the twelve projects reviewed, only one was contracted to provide utility bill analysis.
} 